\title{
REHABILITACIÓN COGNITIVA Y FUNCIONAMIENTO SENSORIAL EN PERSONAS MAYORES CON DETERIORO COGNITIVO LEVE
}

\author{
COGNITIVE REHABILITATION AND SENSORY FUNCTIONING IN OLD PEOPLE WITH MILD \\ COGNITIVE IMPAIRMENT
}

\author{
Angélica María Monsalve Robayo ${ }^{1}$, Valerie Korenfeld Kaplan², Andrea Guarín Caro ${ }^{3}$, Lina María \\ Buitrago Narváez ${ }^{4}$
}

\section{Resumen}

Introducción: Se presentan los resultados obtenidos tras la aplicación de un protocolo de rehabilitación cognitiva basado en el funcionamiento sensorial de personas mayores. Metodología: se trata de un estudio pre-post, en el que un grupo de 4 personas mayores de 55 años con deterioro cognitivo leve participa durante 5 semanas, 3 veces a la semana, 60 minutos cada sesión en un protocolo de rehabilitación cognitiva. Se evalúan los dominios cognitivos, la independencia en actividades instrumentales de la vida diaria y la percepción de la carga del cuidador. Se desarrollan las actividades en cinco etapas propuestas por Ross y Burdick, bajo los principios de integración sensorial. Resultados: Se evidencia el mantenimiento de habilidades como atención y función ejecutiva en dos de los participantes y un mejor desempeño cognitivo en las otras dos personas en áreas como la orientación, la memoria, la percepción, y las operaciones racionales; al igual que en la ejecución de actividades instrumentales de la vida diaria. Discusión: En Colombia son pocos los programas de rehabilitación cognitiva dirigidos a personas mayores, la evidencia muestra que dichos programas generan resultados en la independencia cotidiana, en las relaciones y en el entorno socio-familiar. Se hace necesario aumentar el tiempo de ejecución del protocolo ya que podrían evidenciarse mejores resultados en los dominios anteriormente descritos. Hay una escasa producción académica alrededor del uso de los principios de integración sensorial en los programas de rehabilitación cognitiva, lo que amerita el trabajo por parte de terapeutas ocupacionales en la investigación sobre este tema.

\section{Palabras clave:}

Funcionamiento sensorial; rehabilitación cognitiva; deterioro cognitivo leve; vejez.

\begin{abstract}
Introduction: We present the results obtained after the application of a cognitive rehabilitation protocol based on sensory functioning of elderly. Methodology: this is a pre-post study, in which a group of 4 people over 55 years with mild cognitive impairment participated for 5 weeks, 3 times a week, 60 minutes each session in a cognitive rehabilitation protocol. It assesses the cognitive domains, independence in instrumental activities of daily living and caregiver's perceived burden. Activities are developed in five stages proposed by Ross and Burdick, under the principles of sensory integration. Results: there are evidence about the maintaining of the attention and the executive function in two of the participants and better cognitive performance in the other two people in the áreas of orientation, memory, perception, and rational operations, as in the performance of instrumental activities of daily living. Discussion: In Colombia there are few cognitive rehabilitation programs for older people, the evidence shows that these programs generate results in daily independence,
\end{abstract}

\footnotetext{
${ }^{1}$ Universidad del Rosario. Profesora Programa de Terapia Ocupacional. Cra 24 No 63C-69, Bogotá, Colombia Teléfono: 3474570 ext 562 angelica.monsalve@urosario.edu.co

${ }^{2}$ Estudiante Terapia Ocupacional Universidad del Rosario Cra 24 No 63C-69, Bogotá, Colombia korenfeld.valerie@ur.edu.co

${ }^{3}$ Estudiante Terapia Ocupacional Universidad del Rosario Cra 24 No 63C-69, Bogotá, Colombia. Guarin.andrea@ur.edu.co

${ }^{4}$ Estudiante Terapia Ocupacional Universidad del Rosario Cra 24 No 63C-69, Bogotá, Colombia. Buitrago.lina@ur.edu.co
} 
relationships and the social and family environment. It is necessary to increase the protocol execution time because it could show best performance in the domains described above. There is little academic production around the use of sensory integration principles in cognitive rehabilitation programs, which justifies the work by occupational therapists in research on this topic.

Key words:

Sensory functioning; cognitive rehabilitation; mild cognitive impairment; aging

\section{INTRODUCCIÓN}

El articulo busca presentar los resultados obtenidos en un programa de rehabilitación cognitiva basado en integración sensorial en el cual participó un grupo de personas mayores de 55 años con deterioro cognitivo leve. El interés para el desarrollo de este programa radicó en la necesidad de ofrecer estrategias basadas en evidencia científica que favorecieran el mantenimiento de habilidades cognitivas para el desempeño funcional de actividades de la vida diaria, más aún cuando el crecimiento de la población mayor de 65 años y el riesgo de sufrir trastornos demenciales es evidente en nuestro país. Para lo anterior se utilizaron herramientas de evaluación validadas que midieran el impacto del deterioro en los dominios cognitivos antes y después de la participación en el programa.

Las estadísticas que muestra el documento seguimiento al sector salud en Colombia, aportes al bienestar de la vejez, 2008, establecen que la población colombiana ha venido envejeciendo. Hace 10 años, de cada 100 habitantes de Colombia, cuatro eran mayores de 60 años, en la actualidad se ha aumentado a nueve (Latorre M. 2009). Esto obedece al fenómeno Ilamado transición demográfica, mediante el cual ha venido creciendo la población mayor de 60 años. En países en vías de desarrollo, como Colombia, este fenómeno se da por la disminución de las tasas de natalidad y mortalidad. Lo anterior se evidencia cuando se ve la base de la pirámide poblacional reduciéndose y su punta ensanchándose (Latorre M. 2009).

La agenda sobre Envejecimiento-Colombia siglo XXI afirma que "América Latina está envejeciendo a un ritmo cuatro veces más rápido que el que caracterizó al envejecimiento de los países del llamado primer mundo" (OPS, 2002). Se estima que para el 2025 habrá 8.548.000 personas mayores de 60 años, es decir, el $16,7 \%$ de la población. De este total, el 3,9\% será mayor de 75 años y el $63 \%$ serán mujeres. En Bogotá, este fenómeno se ve reflejado en el aumento de habitantes mayores que representan el 8,99\% del total de personas que viven en la capital (Universidad Del Rosario 2010).

Otro indicador que afecta el proceso de transición demográfica es el relacionado con la esperanza de vida al nacer, en donde Colombia ha venido en aumento al pasar de 67,99 años en el quinquenio 1985-1990 a 74 años proyectado para el quinquenio 2005-2010. Sin embargo, el promedio de Colombia está por debajo de la esperanza de vida de Estados Unidos (78,4 años), Costa Rica (78,9 años) y Chile (78,7años). (Latorre M. 2009).

Este incremento poblacional de personas mayores genera problemas sociales, asistenciales, educativos, familiares y personales que obligan a realizar una planificación de recursos humanos, físicos y financieros para satisfacer necesidades y demandas de servicios para los mayores de 65 años. (Alarcón P. 2006).

La planificación de dichos recursos es prioritaria teniendo en cuenta que los problemas de salud mental, especialmente los trastornos depresivos y las enfermedades neurodegenerativas, entre las que se encuentran las demencias, impactan la calidad de vida personal y familiar. La población de 45 a 64 años que está en proceso de envejecimiento cercano demandará satisfacción de sus necesidades de salud mental, pues es la población con mayor riesgo de iniciar una vejez con estados morbosos mentales, y es la que recibe el mayor impacto de factores psicosociales negativos y posiblemente la menos preparada para afrontarlo de manera adecuada. (Alarcón P. 2006).

El deterioro cognitivo leve (DCL) se comporta como un posible predictor de alteraciones cognitivas progresivas que podrían sugerir el inicio de un cuadro demencial (Petersen R. 2005), por esto, el diagnóstico e intervención a tiempo tiene implicaciones clínicas, familiares y personales importantes.

La demencia es una enfermedad degenerativa que va progresando y ocasiona múltiples alteraciones cognoscitivas, funcionales y sociales. La prevalencia de esta enfermedad, que lleva a incapacidad funcional, laboral o social, depende de la edad y se duplica cada 
cinco años después de los 65 . En personas de 65 años y mayores se ha calculado que la prevalencia es de 1.000 casos por 100.000 habitantes por año. Su correcta identificación es fundamental en la práctica profesional, e incluso necesaria para desarrollar intervenciones efectivas, por lo que algunos expertos creen que debe convertirse en una prioridad de salud pública. Aunque su diagnóstico es relativamente fácil en los estadios moderados o severos, éste se dificulta en etapas tempranas de la enfermedad. (Latorre M. 2009).

Entre las consecuencias de ésta se identifica la discapacidad que genera al verse incrementado el deterioro mental, físico y emocional que trae consigo la necesidad de requerir ayuda de otros para realizar las actividades cotidianas, lo cual hace que la población adquiera una condición de vulnerabilidad. (U. del Rosario 2010).

La política pública colombiana expresa el compromiso con una población que por sus condiciones y características merece especial atención y por tanto se debe abordar con una visión de futuro hacia el proceso de envejecimiento que implica acciones a corto, mediano y largo plazo. (Secretaría Distrital de Salud 2009).

En la actualidad, existen pocos programas que satisfacen las necesidades de salud de esta población, más de la mitad de los mayores en Colombia no tienen seguridad social y el acceso a los servicios asistenciales se ve limitado por las dificultades que impone el sistema. Los programas de salud mental son escasos tanto a nivel gubernamental como a nivel de las empresas promotoras de salud (EPS) (Alarcón P. 2006).

Para atender a dichas necesidades, la presencia de un equipo interdisciplinario ahorraría costos y beneficiaría a la población en el diagnóstico y tratamiento integral. En países como Italia, con más o menos 60 millones de habitantes, existen 500 unidades de diagnóstico; en Colombia con 40 millones de habitantes, se cuenta con tres o cuatro solamente (Latorre M. 2009). Algunas de las actividades que se incluyen en estas unidades no están cubiertas por el Plan Obligatorio de Salud (POS), como es el caso de las evaluaciones neuropsicológicas y los programas de rehabilitación cognitiva que se consideran primordiales para la estimulación permanente de habilidades y el favorecimiento de un desempeño funcional en las actividades de la vida diaria.

\section{Deterioro cognitivo y demencia}

Las fallas en la memoria, los cambios en el comportamiento y una serie de síntomas son parte del diag- nóstico de demencia, sin embargo esta sintomatología puede ser parte de una gran variedad de enfermedades que están catalogadas dentro de las demencias. Su diagnóstico es difícil ya que existen una gran cantidad de aspectos a tener en cuenta, dependiendo de la severidad, el daño cerebral y la funcionalidad de la persona (Gómez-Restrepo C. 2008). Uno de los diagnósticos que se arrojan al realizar una evaluación profunda de los dominios cognitivos, de la esfera social y funcional, es el deterioro cognitivo leve.

Este diagnóstico se define como un estado de transición entre el envejecimiento normal y la demencia, reflejando una situación clínica donde el paciente presenta alteraciones en la memoria con daño cognitivo demostrado, pero sin evidencia de demencia (Armas J .2010); se clasifica en tres tipos: amnésico (alteración específica de la memoria), de múltiples dominios (fallos en la memoria y otras funciones cognitivas), y alteración en un dominio cognitivo diferente a la memoria (alteración importante de otra función cognitiva). (Henao-Arboleda E. 2008).

Para atender a cualquier situación generada por la sintomatología que genera el deterioro cognitivo, es preciso realizar un proceso de rehabilitación cognitiva en el cual las habilidades de procesamiento se estimulen para favorecer un desempeño cotidiano adecuado.

\section{Rehabilitación cognitiva e integración sensorial}

La rehabilitación cognitiva involucra intervenciones diseñadas para cada individuo porque dirige su atención a las dificultades específicas de la persona con deterioro cognitivo o demencia. Se fundamenta en la neuropsicología, la psicología cognitiva y la teoría del aprendizaje. En estas intervenciones se focaliza el trabajo sobre la resignificación de lo cotidiano para el funcionamiento cognitivo y el uso de ayudas compensatorias y estrategias para reducir las demandas sobre la memoria. (Muñoz J. 2008).

La autoiniciación y autorregulación son importantes para seleccionar la intervención adecuada, el conocimiento de la naturaleza y la gravedad de los déficits cognitivos. Las estrategias más comunes en rehabilitación cognitiva son la modificación del ambiente, las estrategias compensatorias y las técnicas de restauración de la función. Las técnicas de aprendizaje especializadas que se desarrollan son el aprendizaje directo, el aprendizaje con errores y el aprendizaje procedimental. (Clare L. 2004) (Clare L. 2003). 
En la rehabilitación cognitiva la sustitución y restauración cobran validez, ya que como lo explica Zangwil (13) citado por Ginarte, en sus investigaciones, es necesario diferenciar entre "entrenamiento directo", compensación y sustitución. Para este autor, la compensación hace referencia a la reorganización de las funciones cognitivas para minimizar una determinada limitación, es espontánea y ocurre sin la participación explícita del paciente. Por sustitución explica la construcción de un método nuevo de respuesta que reemplaza el daño producido por la lesión cerebral. En este caso la intervención busca el aprendizaje de un nuevo tipo de respuestas cuando la lesión afecta a la función original, la búsqueda de nuevas formas o vías para resolver una dificultad. El entrenamiento directo involucra una serie de tareas establecidas para direccionar la función cognitiva que se desea trabajar, especialmente la atención y la memoria.

Muchas personas con este diagnóstico requieren de programas de intervención basados en el reaprendizaje de habilidades dirigidas a la rutina de la vida diaria. Con frecuencia, para la ejecución exitosa de estas tareas, ellas aprenden a utilizar habilidades de manera compensatoria a fin de trabajar en el déficit de las dos funciones mencionadas en el párrafo anterior. La práctica de tareas cotidianas en un ambiente real bajo la supervisión directa del terapeuta y el acompañamiento del cuidador favorecen la identificación de metas con los niveles apropiados de dificultad, adicionalmente se generan preguntas y se hace la retroalimentación necesaria durante la sesión. (Clare L. 2003).

El método mencionado hace parte de la rehabilitación cognitiva, el cual está condicionado por la cantidad de repeticiones que se realicen en el paso de una tarea. Christiansen et al. (1998) declaran que existen pocos estudios controlados que miden la efectividad de técnicas específicas para este tipo de rehabilitación, sin embargo otros estudios (Yevchak A. 2008) (Mahboobin A. 2007) aseguran que la inclusión de una variedad de técnicas compensatorias promueven la conciencia corporal y el descubrimiento personal, las cuales ayudan a mejorar la motivación y la autoestima, resultado que permite observar de alguna forma el desempeño cognitivo.

En este sentido la innovación en la implementación de estrategias que evolucionen hacia el uso de los sentidos como estructuras que permiten la identificación de estímulos para una posterior integración de los mismos en escenarios cotidianos cobra valor, sobre todo cuando se evidencian dificultades en la forma en que las personas con demencia procesan los estímulos. Estu- dios como el realizado por Rizzo et al. en el año 2000, muestran cómo las dificultades en el funcionamiento sensorial limitan el procesamiento de las funciones superiores más altas y disminuyen la capacidad de responder cognitivamente a tareas de la vida diaria como leer, seguir una ruta, reconocer o localizar objetos.

Las respuestas adaptativas son el producto final de un proceso en el cual el sistema nervioso central toma la información, la interpreta y produce una acción significativa, las respuestas pueden ser afectivas, motoras, fisiológicas o funcionales. Sin embargo estas respuestas pueden alterarse por los déficits cognitivos que disminuyen la capacidad de atención, lo cual afecta la velocidad de procesamiento de la integración sensorial en este colectivo (Mahboobin A. 2007).

La integración sensorial busca cambiar e influenciar el desarrollo de funciones neurológicas básicas a través del uso de experiencias sensoriales organizadas por etapas. Mildred Ross, 1981, adaptó el uso de la teoría a personas con trastornos degenerativos estableciendo los beneficios de cada una de las etapas en relación con la estimulación de centros neurológicos especializados.

La influencia del funcionamiento sensorial sobre el procesamiento de las funciones superiores constituye un factor relevante para considerarse en el proceso de rehabilitación cognitiva, por tanto, la participación de las estructuras cerebrales de la corteza en la discriminación y relación de la información para ser traducida en acción, constituye un fundamento propicio para la aplicación de la técnica de integración sensorial (Monsalve A. 2009).

Elementos como el aprendizaje, el ejercicio y el entrenamiento cognitivo parecen constituirse en transversales de la rehabilitación exitosa al producir resultados efectivos en los sujetos con demencia, ya que permiten generar respuestas adaptativas que se evidencian en el mantenimiento de habilidades funcionales donde el sujeto y el cuidador se involucran activamente en el proceso terapéutico.

Existe una necesidad generalizada en los estudios revisados de medir la efectividad de los programas de rehabilitación cognitiva, incluyendo la valoración inicial de los sujetos y el posterior desempeño.

Los resultados de los estudios (Ginarte Y. 2002) (Christiansen C. 1998) (Robichaud L. 1990) (Corcoran M. 1987) donde se utilizan programas de rehabilitación cognitiva y específicamente el enfoque de integración sensorial, manifiestan un progreso significativo en las habilidades para participar en actividades socio-familiares, mejoría en habilidades como la orientación, la regulación, el recuerdo y el lenguaje. También se redu- 
ce la agitación, se promueve la conciencia corporal y se estabiliza el desempeño cognitivo y funcional.

La integración sensorial es el proceso neurológico que organiza las sensaciones propioceptivas, vestibulares y táctiles para el uso efectivo del cuerpo en un ambiente determinado (Ayres J. 1971). Este enfoque se fundamenta en cinco principios que sustentan los beneficios que se reflejan en el sistema mente-cerebrocuerpo (Ayres J. 1989), evidenciando la interdependencia entre las funciones del mismo.

El primer principio se refiere a la capacidad que tiene el cerebro para adaptarse y moldearse en las diferentes etapas del ciclo vital, lo cual es reportado por Mattson (2001) y Levin (2006), quienes hallaron la existencia de cambios en el cerebro incluso en organismos maduros y geriátricos con lesiones cerebrales, tras someterse a ambientes enriquecidos y estudios de neuroplasticidad e imagenología cerebral.

El segundo principio explica que la integración sensorial tiene un proceso por etapas en espiral que se produce gracias a la estimulación que se brinda a ciertos niveles cerebrales subcorticales, favoreciendo la maduración o mejor funcionamiento de habilidades que permiten que el cerebro trabaje como un sistema integrado.

El tercer principio muestra la integración de las funciones cerebrales, lo cual es explicado por Jean Ayres (1971) al referir que las estructuras subcorticales evolucionan y maduran antes que las estructuras corticales, evidenciándose la interdependencia entre dichas funciones y la experiencia sensorio-motora (Ayres J. 1971).

El cuarto principio indica que la integración sensorial requiere de la interacción y la adaptación en diferentes entornos, lo cual se refleja en el aprendizaje que se obtiene del auto reconocimiento de las experiencias vividas previamente (Ayres J. 1989).

El quinto principio se fundamenta en la capacidad que tienen las personas para motivarse y orientar sus acciones hacia la participación en actividades sensoriales y motoras (Ayres J. 1989).

Existen otros enfoques que aportan a los fundamentos de la integración sensorial, como lo son el de control motor y el cognitivo perceptual. El primero de ellos explica la capacidad que tienen los sujetos para utilizar el propio cuerpo de manera efectiva durante el desempeño de una ocupación, lo cual hace que algunos patrones de comportamiento motor como la fuerza, la maduración del sistema nervioso central, las propiedades de los objetos que se manipulan y la velocidad de acción necesaria para la tarea cambien. (Kielhofner G. 2006). Dentro de éste se incluyen dos componentes: la generación y la coordinación de patrones de movimiento de la cabeza, las extremidades y el tronco, y el mantenimiento del equilibrio durante el desempeño de la tarea.

Por otro lado, el enfoque cognitivo perceptual propuesto por Toglia y Abreu (1987), explica que la percepción es un proceso dinámico que implica "la detección y el análisis sensorial, la formulación de hipótesis y la decisión de una respuesta", y en este sentido se trabaja sobre la comprensión de la capacidad de procesamiento de la información del cerebro y el daño que sufre en los casos de lesión. La cognición por su parte, requiere de la atención, la memoria, la planificación, la reflexión y la solución adaptativa de problemas. (Wilson B. 2002).

A partir de lo anterior se hace necesario implementar un programa que permita en personas con deterioro cognitivo o estadios iniciales de demencia: alertar los sistemas sensoriales, favorecer el uso del movimiento como organizador de estímulos, promover la integración perceptual, el control motor y la comunicación interhemisférica, estimular habilidades cognitivas, favorecer la interacción grupal y la percepción de sí mismo; para así enlentecer el proceso de deterioro y favorecer la adaptación en el entorno familiar y social.

\section{Metodología}

Se trata de un estudio de tipo cuasi-experimental, en donde no hay aleatorización de los sujetos y no existe un grupo control propiamente dicho (Rodríguez M. 2007). El diseño más habitual de este tipo de investigación es el estudio antes-después (o pre-post) de un solo grupo. En el estudio se midió y comparó diferentes variables antes y después de la exposición del sujeto a la intervención. Se acudió al muestreo intencional o propositivo, que es aquel en el que los sujetos de la muestra no son elegidos siguiendo las leyes del azar, sino de manera intencional (Ruiz J. 1999).

La intervención se desarrolló a través de un protocolo de rehabilitación cognitiva que se implementó con un grupo de personas mayores de 55 años con deterioro cognitivo leve, quienes fueron remitidos por el servicio de neuropsicología del Hospital Universitario Mayor al laboratorio de terapia ocupacional de la Sede Quinta Mutis de la Universidad del Rosario, ubicada en Bogotá. La población poseía conocimientos de lectura y escritura y contaba con una red de apoyo primario; firmaron un consentimiento informado el cual fue avalado por el Comité de Ética en Investigación de la 
Universidad. En la tabla 1 se presentan los datos sociodemográficos de la población participante.

Tabla 1. Datos socio-demográficos de la población

\begin{tabular}{|c|c|c|c|c|}
\hline Paciente & Genero & Edad & Nivel educativo & Ocupación \\
\hline 1 & Femenino & 70 & $\begin{array}{c}\text { Secundaria } \\
\text { incompleta }\end{array}$ & Pensionada \\
\hline 2 & Masculino & 69 & $\begin{array}{c}\text { Técnica } \\
\text { (contaduría) }\end{array}$ & Pensionado \\
\hline 3 & Femenino & 70 & $\begin{array}{c}\text { Secundaria } \\
\text { completa }\end{array}$ & Pensionada \\
\hline 4 & Femenino & 79 & $\begin{array}{c}\text { Secundaria } \\
\text { incompleta }\end{array}$ & Hogar \\
\hline
\end{tabular}

Se utilizaron seis evaluaciones al inicio y al final del programa para conocer los cambios en los dominios cognitivos, en la independencia en actividades instrumentales de la vida diaria y en la carga percibida por el cuidador. Dichas evaluaciones fueron: Mini-Mental
State Examination (MMSE) de Folstein, Trail Making Test (forma A y B) TMT, Índice de Lawton y Brody, Loewenstein Occupational Therapy Cognitive Assessment (LOTCA) y la Entrevista de carga del cuidador de Zarit.

El procedimiento del estudio incluyó: la realización de una anamnesis ocupacional diligenciada a través de un formato de registro; la evaluación de los dominios cognitivos, la carga percibida del cuidador y las actividades instrumentales de la vida diaria; la aplicación del protocolo de rehabilitación con una intensidad de tres veces a la semana durante 5 semanas, 60 minutos cada sesión y la evaluación posterior de los mismos elementos enunciados anteriormente. El análisis de resultados obtenidos en las diferentes evaluaciones se realizó a través de análisis de frecuencias.

La organización y planeación de una sesión de trabajo para la aplicación del protocolo de rehabilitación se fundamentó en los principios de integración sensorial y constó de cinco etapas ya establecidas por Ross y Burdick (1981), que buscaba producir estimulación progresiva en diferentes canales y con conexiones de centros neurológicos cada vez más altos para favorecer la producción de respuestas adaptativas a través de la acción (Tabla 2).

Tabla 2. Etapas del protocolo de rehabilitación cognitiva basado en los principios de integración sensorial

\begin{tabular}{|c|c|c|}
\hline & Descripción & Recursos \\
\hline$\frac{\pi}{\frac{\pi}{\pi}}$ & $\begin{array}{l}\text { Alertar los sistemas sensoriales, activar los sistemas } \\
\text { reticulares y nervios craneales como el II, III, IV y VI; } \\
\text { por medio de la estimulación de sentidos como el } \\
\text { olfato, el tacto y la audición. }\end{array}$ & Estímulos olfativos, táctiles y auditivos. \\
\hline 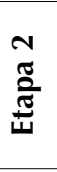 & $\begin{array}{c}\text { Proveer estímulos sensoriales que favorezcan el uso } \\
\text { del movimiento como organizador de dichos } \\
\text { estímulos }\end{array}$ & $\begin{array}{l}\text { Movimientos organizados de grandes grupos } \\
\text { musculares a nivel proximal para lograr control } \\
\text { postural y transferencia a las cinturas escapular y } \\
\text { pélvica (27). Estímulos posturales y del equilibrio. }\end{array}$ \\
\hline$\frac{n}{\stackrel{n}{\pi}}$ & $\begin{array}{c}\text { Promover la integración perceptual, el control motor } \\
\text { y la comunicación inter-hemisférica. }\end{array}$ & $\begin{array}{c}\text { Actividades de planeación, secuenciación, integración } \\
\text { bilateral, lateralidad, solución de problemas, } \\
\text { categorización y reconocimiento }\end{array}$ \\
\hline 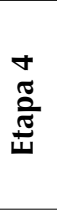 & $\begin{array}{l}\text { Trabajar los dominios cognitivos teniendo como base } \\
\text { la reestructuración del esquema corporal y la esti- } \\
\text { mulación de la médula espinal hasta los niveles más } \\
\text { altos de la corteza pasando por los nervios craneales, } \\
\text { el tallo cerebral y el diencéfalo }\end{array}$ & $\begin{array}{l}\text { Estímulos cognitivos, que promuevan la memoria, la } \\
\text { atención, el lenguaje, las gnosias, las praxias, las fun- } \\
\text { ciones ejecutivas, el cálculo y la orientación. }\end{array}$ \\
\hline 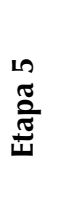 & $\begin{array}{c}\text { Favorecer la interacción grupal y la percepción de sí } \\
\text { mismo para así fijar las sensaciones que se han } \\
\text { proporcionado en etapas anteriores y lograr estimular } \\
\text { las estructuras del sistema límbico y la } \\
\text { formación reticular. }\end{array}$ & $\begin{array}{l}\text { Espacio para las relaciones interpersonales y la } \\
\text { interacción grupal. Estímulo gustativo. }\end{array}$ \\
\hline
\end{tabular}




\section{Resultados}

El protocolo se socializó con 9 personas que cumplían con los criterios de inclusión del estudio, pero de éstos, solo 4 permanecieron hasta el final de su aplicación, cumpliendo con la frecuencia e intensidad de las sesiones terapéuticas.

Los resultados obtenidos en la prueba LOTCA (tabla 3) que determina el desempeño en las habilidades de orientación, percepción, organización visomotora y operaciones racionales muestran en general una mejoría en el grupo. En cuanto a la habilidad de orientación se evidencia en el paciente uno, un aumento del $62,5 \%$ en el desempeño de la prueba, mientras que en los demás pacientes el puntaje obtenido fue igual al del inicio del estudio. En percepción, el paciente uno mejoró en un $16,7 \%$ y el paciente tres en un $4,17 \%$, el paciente cuatro por su lado disminuyó su desempeño en un 8,33\%. En organización visomotora tres de los cuatro pacientes mejoraron su desempeño, el paciente dos en un 10,7\% y el paciente tres y cuatro en un $7,1 \%$. Finalmente, la habilidad de operaciones racionales es la que muestra un mejor desempeño en los pacientes después de participar en el protocolo; solo el paciente tres mantuvo su desempeño, mientras que los pacientes uno, dos y cuatro mejoraron en un $21,7 \%, 30,4 \%$ y $17,4 \%$ respectivamente.

Tabla 3. Puntajes obtenidos en el LOTCA

\begin{tabular}{|c|c|c|c|c|c|c|c|c|}
\hline \multirow{2}{*}{ Paciente } & \multicolumn{2}{|c|}{ Orientación } & \multicolumn{2}{|c|}{ Percepción } & \multicolumn{2}{c|}{$\begin{array}{c}\text { Organización } \\
\text { visomotora }\end{array}$} & \multicolumn{2}{c|}{$\begin{array}{c}\text { Operaciones } \\
\text { racionales }\end{array}$} \\
\cline { 2 - 9 } & Pre & Post & Pre & Post & Pre & Post & Pre & Post \\
\hline 1 & $2 / 8$ & $7 / 8$ & $17 / 24$ & $21 / 24$ & $8 / 28$ & $8 / 28$ & $5 / 23$ & $10 / 23$ \\
\hline 2 & $8 / 8$ & $8 / 8$ & $23 / 24$ & $23 / 24$ & $21 / 28$ & $24 / 28$ & $13 / 23$ & $20 / 23$ \\
\hline 3 & $7 / 8$ & $7 / 8$ & $22 / 24$ & $23 / 24$ & $16 / 28$ & $18 / 28$ & $15 / 23$ & $15 / 23$ \\
\hline 4 & $8 / 8$ & $8 / 8$ & $23 / 23$ & $21 / 21$ & $18 / 28$ & $20 / 28$ & $8 / 23$ & $12 / 23$ \\
\hline
\end{tabular}

Otro resultado del estudio es el arrojado por el MiniMental State Examination (MMSE) de Folstein, que explora la orientación espacial y temporal, memoria inmediata y remota, lenguaje, atención, cálculo, praxis ideacional y constructiva. Los resultados de dicha prueba (Tabla 4) muestran que dos de los participantes mantuvieron los resultados del pretest y los otros dos presentaron en promedio una mejoría del $8,66 \%$. Los resultados del Trail Making Test (forma A y B) (Tabla 4), el cual provee información de búsqueda visual, escaneo, velocidad de procesamiento y funciones ejecutivas, evidencian que en el TMT-A solo el paciente uno excedió el tiempo de la prueba en comparación con la evaluación inicial, considerando los dos resultados como deficitarios en la interpretación de la prueba, ya que superó los 78 segundos. El paciente dos mantuvo el tiempo de ejecución, los pacientes tres y cuatro lo disminuyeron significativamente, lo que indica mejoría en la capacidad atencional. En el TMT-B, el paciente uno tuvo dificultad para realizar la prueba en los dos momentos, el paciente dos mantuvo el tiempo de ejecución y los pacientes tres y cuatro aumentaron el tiempo de ejecución, excediendo el estipulado por la prueba para identificar dificultades en la función ejecutiva y en la atención dividida ( $>273 \mathrm{seg}$ ).

Tabla 4. Puntajes obtenidos en el MMSE y TMT

\begin{tabular}{|c|c|c|c|c|c|c|}
\hline \multirow{2}{*}{ Paciente } & \multicolumn{2}{|c|}{ MMSE } & \multicolumn{2}{|c|}{ TMTA } & \multicolumn{2}{c|}{ TMTB } \\
\cline { 2 - 6 } & Pre & Post & Pre & Post & Pre & Post \\
\hline 1 & $22 / 30$ & $25 / 30$ & 240 seg/>78seg & 390 seg/>78seg & NA & NA \\
\hline 2 & $29 / 30$ & $29 / 30$ & 70 seg/>78seg & 70 seg/>78seg & 260 seg/>273seg & 260 seg/>273seg \\
\hline 3 & $27 / 30$ & $27 / 30$ & $201 \mathrm{seg} />78 s e g$ & 50 seg/>78seg & 300 seg/>273seg & 320 seg/>273seg \\
\hline 4 & $28 / 30$ & $30 / 30$ & $93 \mathrm{seg} />78 \mathrm{seg}$ & $48 \mathrm{seg} />78 \mathrm{seg}$ & $393 \mathrm{seg} />273 \mathrm{seg}$ & $443 \mathrm{seg} />273 \mathrm{seg}$ \\
\hline
\end{tabular}


Para la evaluación de las actividades instrumentales de la vida diaria se utilizó el Índice de Lawton y Brody, el cual arroja una puntuación máxima de 30 (dependencia total) y una mínima de 8 (independencia total); los resultados obtenidos muestran el mantenimiento de la independencia en dos de las personas y un aumento en la misma en el paciente uno y cuatro.

Tabla 5. Puntajes obtenidos en Independencia en AIVD

\begin{tabular}{|c|c|c|}
\hline \multirow{2}{*}{ Paciente } & \multicolumn{2}{|c|}{$\begin{array}{c}\text { Independencia en actividades } \\
\text { instrumentales de la vida diaria (AIVD) }\end{array}$} \\
\cline { 2 - 3 } & Pre & Post \\
\hline 1 & $16 / 30$ & $11 / 30$ \\
\hline 2 & $10 / 30$ & $10 / 30$ \\
\hline 3 & $8 / 30$ & $8 / 30$ \\
\hline 4 & $19 / 30$ & $14 / 30$ \\
\hline
\end{tabular}

Finalmente, en la aplicación de la escala de sobrecarga del cuidador de Zarit, la cual indica que un puntaje mayor a 55 representa sobrecarga intensa y menos de 47 que no hay sobrecarga, se encontró que ninguno de los familiares de los integrantes del grupo percibe esta sensación, lo que podría explicarse de alguna manera por la autonomía en la realización de las actividades de la vida diaria y la distribución de tareas entre los familiares para asumir responsabilidades compartidas.

Tabla 6. Puntajes obtenidos en la escala de Zarit

\begin{tabular}{|c|c|c|}
\hline \multirow{2}{*}{ Paciente } & \multicolumn{2}{|c|}{ Escala de Zarit } \\
\cline { 2 - 3 } & Pre & Post \\
\hline 1 & $20 />55$ & $20 />55$ \\
\hline 2 & $47 />55$ & $45 />55$ \\
\hline 3 & $23 />55$ & $21 />55$ \\
\hline 4 & $38 />55$ & $49 />55$ \\
\hline
\end{tabular}

\section{DISCUSIÓN}

Algunos estudios hacen una revisión profunda sobre los principios y fundamentos en el campo de la rehabilitación cognitiva y se asegura que la recuperación luego de un proceso de rehabilitación no se debe producir solo a nivel físico sino que además debe impactar el contexto socio-familiar donde se desenvuelve la persona (Wilson B. 2002) (Ginarte Y. 2002) (Christiansen C. 1998). Adicionalmente, se identifica que los programas que trabajan habilidades de memoria son reconocidos como buenas prácticas en demencia.

Los mismos estudios, definen este proceso como la aplicación de procedimientos, técnicas y la utilización de apoyos que ayudan a la persona a retomar de manera segura, productiva e independiente sus actividades diarias.

En cuanto a la importancia de los principios de la integración sensorial y su influencia en el desempeño cognitivo, Ross y Burdick (1981), Robichaud (1994), Corcoran y Barret, (1987), Roberts King-Thomas y Boccia (2007), reportan que ésta cambia e influencia el desarrollo de las funciones neurológicas a través de experiencias sensoriales organizadas por etapas, fundamentadas en la plasticidad cerebral, en la evolución de la integración sensorial, en el trabajo integrado de las funciones subcorticales y corticales, en la interacción adaptativa y en la orientación interna del sujeto para participar en actividades sensorio-motoras.

Algunos estudios (Ginarte Y. 2002), (Christiansen C. 1998) argumentan que el uso de este enfoque permite la disminución de comportamientos disruptivos o agresivos y se mejora el desempeño en actividades de la vida diaria, siempre y cuando la eficacia de la intervención esté dada por el funcionamiento social y el comportamiento orientado a la tarea.

Es evidente la escasa publicación de resultados en torno al uso de este enfoque en personas mayores con deterioro cognitivo, esto podría explicarse por los desarrollos y avances que ha tenido desde otras condiciones patológicas en otras poblaciones. Lo anterior representa una necesidad para los profesionales en Terapia Ocupacional que trabajan y estudian la efectividad de las intervenciones en función del desempeño cotidiano de la población.

\section{RefERENCIAS BibliográfICAS}

Abreu B.; Toglia J. Cognitive rehabilitation: a model for occupational therapy. American journal of occupational therapy 1987; 41: 439- 448.

Alarcón P. Introducción a la Psiquiatría Geriátrica. En: Manual Básico de Psiquiatría Geriátrica. Capítulo 1. Risaralda. 2006.

Armas J., Valdés S., Mena Y. Factores predictores clínicos y neuropsicológicos de la conversión del deterioro cognitivo leve 
a la enfermedad de alzheimer. 11o Congreso Virtual de Psiquiatría. 2010.

Ayres J. Characteristics of types of sensory integrative dysfunction. American Journal of Occupational Therapy. 1971, 25: 329-334.

Ayres J. Sensory Integration and Praxis Tests Manual. Los Angeles: Western Psychological Service. 1989.

Christiansen C., Abreu B., Ottenbacher K., Huffman K., Masel B., Culpepper R. Task Performance in virtual environments used for cognitive rehabilitation after traumatic brain injury. Arch Phys Med Rehabil 1998; 79: 888-92.

Clare L., Woods R. Cognitive training and cognitive rehabilitation for people with early - stage Alzheimer's disease: a review (2004). Neuropsychological Rehabilitation; 14 (4): 385-401.

Clare L. Cognitive training and cognitive rehabilitation for people with early-stage dementia (2003). Reviews in clinical Gerontology; 23: 75.

Corcoran M. Using sensory integration principles with regressed elderly patients. En: Sensory Integrative Approaches in Occupational Therapy. New York. The Haworth Press. 1987.

Ginarte Y. Rehabilitación cognitiva. Aspectos teóricos y metodológicos. Rev Neurol 2002; 35 (9): 870-876.

Gómez-Restrepo C., Hernández G., Rojas A., Santacruz H., Uribe M. Fundamentos de Psiquiatría Clínica: Niños, Adolescentes y Adultos. CEJA. Bogotá - Colombia. 2008.

Henao-Arboleda E., Aguirre-Acevedo D., Muñoz C., Pineda D., Lopera F. (2008). Prevalencia de deterioro cognitivo leve de tipo amnésico en una población colombiana. REV NEUROL; 46 (12): 709-713.

Kielhofner G. Fundamentos conceptuales de la Terapia Ocupacional. 3a. Edición. Editorial Panamericana. 2006.

Latorre M., Cobos C., Beltrán H. Documento Aportes al Bienestar en la Vejez. Seguimiento al sector salud en Colombia. Bogotá. 2009.

Levin H. Neuroplasticity and brain imaging research: Implications for Rehabilitation. Archive.

Mahboobin A., Loughlin P., Redfern M. A model-based approach to attention and sensory integration in postural control of older adults. Neuroscience letters. 2007; 429:147-159.

Mattson M., Camandola S. "NF - KB in neuronal plasticity and neurodegenerative disorders". The Journal of Clinical Investigation. 107, No 3 (2001): 247-254.

Monsalve A., Rozo C. Integración sensorial y demencia tipo Alzheimer: principios y métodos para la rehabilitación. Rev. Colomb. Psiquiat., vol 38. No 4. 2009.

Muñoz J., Tirapu J. Rehabilitación neuropsicológica. Editorial SíNTESIS. Madrid. 2008.

ORGANIZACIÓN PANAMERICANA DE LA SALUD. Agenda sobre Envejecimiento Colombia Siglo XXI. Bogotá. 2002.
Petersen R. Mild cognitive Impairment as a clinical entity and treatment target. Arch Neurol 2005; 62: 1160.

Rizzo M., Anderson S., Dawson J., Nawrot M. Vision and cognition in Alzheimer's disease. Neuropsychologia. 2000; 38:11571169.

Roberts J., King-Thomas L., Boccia M. Behavioural indexes of the efficacy of sensory integration therapy. The American Journal of Occupational Therapy. 2007, 61: 555-562.

Robichaud L., Hebert R., Desrosiers J. Efficacy of a Sensory Integration Program on Bechaviors of Inpatients with Dementia. American Journal of Occupational Therapy. 355-360. 1990.

Rodríguez M., Pérez S., Sordo L., Fernández M. Cómo elaborar un protocolo de investigación en salud. Med Clin (Barc). 2007; 129(8):299-302.

Ross M., Burdick D. Sensory Integration: A training manual for therapists and teachers for regressed, psychiatric and geriatric patient group. Slack Incorporated. New Jersey. 1981.

Ruiz J. Metodología de la Investigación Educativa. Universidad de Deusto. Bilbao, España. 1999.

Secretaría Distrital de Salud. Lineamientos Plan de Intervenciones Colectivas. Promoción de la Calidad de Vida y Salud de Envejecimiento y Vejez. 2009.

Universidad Del Rosario. Asistencia al adulto mayor: ¿caridad o derecho? Facultad de Jurisprudencia. 14 fascículo interactivo. Universidad, Ciencia y Desarrollo. Programa de Divulgación Científica. Tomo V. 2010.

Wilson B. Towards a comprehensive model of cognitive rehabilitation (2002). Neuropsychological rehabilitation; 12 (2): 97-110.

Yevchak A., Loeb S., Fick D. Promoting Cognitive Health and Vitality: A Review of Clinical Implications. Geriatr Nurs 2008; 29: 302-310. 